\title{
Current Insights on the Impact of Gamma-Hydroxybutyrate (GHB) Abuse
}

\author{
Emma Tay $\mathbb{D}^{\prime}$, Wing Kwan Winky Lo², Bridin Murnion $\mathbb{D}^{3}$ \\ 'Drug Health Services, Western Sydney Local Health District, Sydney, NSW, Australia; ${ }^{2}$ Faculty of Medicine, University of New South Wales, Sydney, \\ NSW, Australia; ${ }^{3}$ Faculty of Medicine and Health, University of Sydney, Sydney, NSW, Australia
}

Correspondence: Bridin Murnion, Drug Health Services, Western Sydney Local Health District, 4A Fleet Street, North Parramatta, NSW, 2I5I, Australia, Tel +6I 29840 3888, Fax +6I 29840 3927, Email bridin.murnion@sydney.edu.au

\begin{abstract}
Recreational gamma-hydroxybutyrate (GHB) use, although less common than other substance use, is increasingly recognised and is over-represented in emergency toxicology presentations. This narrative review summarizes GHB pharmacology, current patterns of use, potential harms and management of GHB toxicity and withdrawal. There is a complex interplay between GHB and GABA as GHB is both a prodrug and metabolite of GABA and GHB activates both GHB and GABA receptors. GHB is rapidly absorbed, with effects seen within minutes of ingestion. Metabolism is non-linear at higher doses. While GHB is listed as a controlled substance, its precursor's gamma-butyrolactone (GBL) and 1,4-butanediol (1,4-BD) are easily available as both have industrial applications. National surveys indicate low rates of GHB use, with identification of high-risk populations in men who have sex with men and polysubstance users. GHB is one of the three drugs most commonly used in chemsex. GHB is often co-ingested with other interacting psychoactive substances. Acute toxicity is dose-dependent, and management is supportive care. Withdrawal management is generally with benzodiazepines with addition of baclofen for more severe withdrawal. Barbiturates may have a role. Titration and tapering of pharmaceutical GHB is commonly used in the Netherlands. Complicated withdrawal with delirium may require intensive care and treatment with intravenous sedation. There are high rates of relapse after withdrawal and medications for longerterm management are currently being investigated. Chronic use is associated with poorer mental, physical and sexual health, social dysfunction and poor work performance. Laboratory detection is complicated as GHB is an endogenous substance with a short halflife, and therefore not often routinely assayed in the clinical setting. Future research should focus on improving GHB detection and management of GHB withdrawal and dependence. Interventions specific for high-risk groups should be developed and assessed.
\end{abstract}

Keywords: gamma-hydroxybutyrate, gamma-butyrolactone, 1, 4-butanediol, use, harms, management

\section{Plain Language Summary}

In this review, we describe the chemistry, use and harms of gamma-hydroxybutyrate (GHB). Treatment for GHB withdrawal, overdose and dependence is also discussed.

- GHB occurs naturally in the human brain.

- GHB from external sources can also be ingested recreationally for euphoria.

- Gamma-butyrolactone (GBL) and 1,4-butanediol (1,4-BD) are readily available as industrial solvents and can be consumed and converted by the body to GHB.

- Detecting ingested GHB in blood, urine or hair is complicated because it is a naturally occurring substance and is rapidly broken down in the body.

- GHB use is uncommon compared to other drugs, but overdose can have severe consequences.

- GHB acts as an inhibitory chemical in the brain, and people can become comatose when they overdose. This may require mechanical breathing support.

- People can also become dependent on GHB and in severe cases, people may need to use GHB every few hours.

- Apart from overdose and dependence, a range of different issues are reported with GHB use. These include driving under the influence, sleep disruption, and problems with mental and sexual health. 
- Withdrawal from GHB is similar to alcohol withdrawal but can become severe quite rapidly. Treatment is primarily with sedatives and supportive care.

- Improving treatment of GHB dependence is currently being investigated.

- Future research should also focus on working with groups at high risk of GHB use and harm.

\section{Introduction}

Gamma-hydroxybutyrate (GHB) is an endogenous neurochemical. Ingestion of supra-physiological amounts can cause euphoria. This recreational use of GHB is gaining recognition and the associated acute and chronic harms can cause significant morbidity and mortality. ${ }^{1,2}$ In this narrative review, a summary of the pharmacology of GHB will be provided. The epidemiology of GHB use will be described with a discussion of identification and management of acute and chronic harms.

\section{Methods}

PubMed, medline, and google scholar were searched from 1 January 2010 to 8 December 2021. Search terms used were GHB, gamma hydroxy butyrate, GBL, gamma-butyrolactone, 1,4-BD and 1,4-butanediol combined with use, dependence, epidemiology, harms, withdrawal, treatment, regulation, chemsex, driving, detection or analysis. Reference lists of relevant articles were searched for articles relevant to the narrative review. The search was limited to articles in English language. For country-related prevalence information, the most recently published World Health Organisation, European, US, Australian and Dutch country-level drug use reports published in English were included.

\section{Pharmacology}

Initially developed as a gamma-aminobutyric acid (GABA) analogue in the mid-20th century, GHB has subsequently been identified to exist endogenously in the mammalian central nervous system where it is associated with thermoregulation, sleep cycle, sympathetic tone and learning. ${ }^{3}$ It is also found in peripheral tissues and other organs such as the heart, kidneys, muscle and brown fat. ${ }^{4}$ It has also been identified as present in wine, beef, citrus fruits, non-alcoholic carbonated beverages and most animals, albeit in small amounts. ${ }^{5,6}$ Endogenous GHB is synthesized from GABA, which originates from glutamate. ${ }^{7}$ GHB is in part degraded via the Krebs cycle through formation of succinic semialdehyde and succinic acid, and in part metabolised to GABA via GABA transaminase (Figure 1). This makes GHB both a prodrug and metabolite of GABA. ${ }^{7}$

In vivo, the prodrugs gamma-butyrolactone (GBL) and 1,4-butanediol (1,4-BD) are rapidly metabolised to GHB (Figure 1). GBL is catalysed by the enzyme lactonase. 1,4-BD is metabolised by alcohol dehydrogenase to 4-hydroxybutyraldehyde, which is then converted to GHB by aldehyde dehydrogenase. ${ }^{7}$ Alcohol dehydrogenase can become saturated with co-ingested alcohol (Figure 1), thus delaying biotransformation of 1,4-BD until alcohol is metabolised, with delayed onset of GHB toxicity. ${ }^{8}$

GHB binds with high affinity to GHB-specific receptors and to the $\alpha 4 \beta \delta$ subtype of the $\mathrm{GABA}_{\mathrm{A}}$ receptor, and with lower affinity to $\mathrm{GABA}_{\mathrm{B}}$ receptors in the brain. ${ }^{4,9} \mathrm{GHB}$ receptors are located both pre- and post-synaptically and may moderate neurotransmitter release. ${ }^{4}$ Unlike exogenous GHB that produces much higher concentrations, GHB at physiological concentrations is thought to activate GHB and the $\alpha 4 \beta \delta \mathrm{GABA}_{\mathrm{A}}$ receptors but not the $\mathrm{GABA}_{\mathrm{B}}$ receptors. These are activated at supra-physiological concentrations of GHB. ${ }^{4}$

Clinical effects of GHB were initially believed to be mediated through modulation of the GABA inhibitory neurotransmission system, ${ }^{3}$ although more recent reports have suggested the possibility of a mixed GABA/GHB receptor mechanism of action in vivo, ${ }^{4}$ further emphasizing these two closely linked systems. Additional effects of GHB include modulating dopamine activity and release, serotonin systems, and opioid systems. ${ }^{7}$

Recreational GHB is usually found as a colourless, odourless aqueous preparation. When ingested, it is rapidly absorbed orally, with onset of action within 15 to 30 minutes, and peak serum concentrations occurring between 25 minutes and one hour after ingestion. ${ }^{10,11} \mathrm{GHB}$ easily crosses the blood-brain barrier and monocarboxylate transporters may facilitate that. $^{7}$ Exogenously administered GHB undergoes extensive hepatic metabolism, with only a minority (less than 2\%) excreted unchanged in urine. ${ }^{7,10}$ The elimination half-life of GHB is short at around 40 minutes, and follows first-order kinetics at moderate doses. ${ }^{11}$ Elimination pattern at higher doses is non-linear due to saturable metabolic pathways. ${ }^{7}$ This is further 


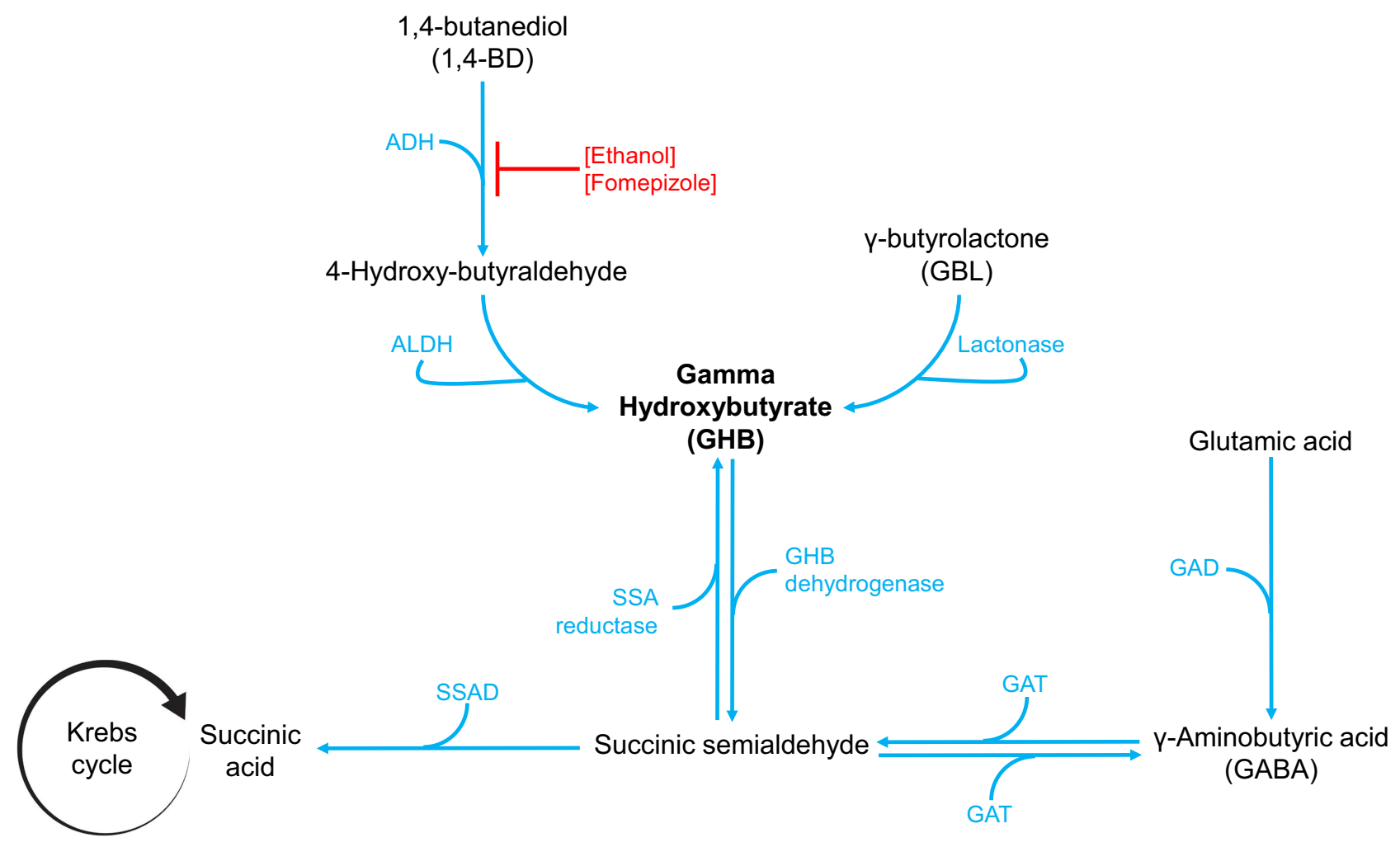

Figure I Pathways of GHB synthesis and metabolism. ADH is inhibited by alcohol and fomepizole.

Abbreviations: ADH, alcohol dehydrogenase; ALDH, aldehyde dehydrogenase; GAD, glutamic acid decarboxylase; GAT, GABA transaminase; SSA, succinic semialdehyde; SSAD, succinic semialdehyde dehydrogenase.

supported by a non-linear dose-exposure relationship in studies in healthy subjects, with plasma concentration at higher doses rising disproportionately compared to lower doses. ${ }^{11}$

GBL and 1,4-BD are similarly well absorbed orally. In one study of eight healthy volunteers, ingestion of 1,4-BD led to detectable GHB concentrations within five minutes in the majority of the subjects. ${ }^{12}$

\section{Early Use}

The use of exogenous GHB was first described in the 1960s when it was manufactured and trialled as a new anaesthetic agent. ${ }^{13}$ Its precursors, GBL and 1,4-BD were discovered earlier, with the former's anaesthetic property initially reported in animal model studies in $1947 .{ }^{14}$ 1,4-BD has been available since the late 19th century and importantly is still commercially available as an industrial solvent. ${ }^{15}$ GHB and GBL were not further established as general anaesthetic agents as they lack analgesic and muscle relaxant properties. ${ }^{15}$ In addition, multiple adverse effects are associated with their use, including random myoclonic movements, bradycardia, nausea, vomiting, hallucinations, respiratory depression, and apnea. ${ }^{5,15}$ Recreational use of GHB gained popularity through the 1980 s to 1990 s with body builders using it for its apparent anabolic effect in stimulating growth hormone. ${ }^{7}$ Others used GHB seeking the purported euphoric and sexual performance enhancing effects. ${ }^{7}$ GHB has also gained some notoriety as a "date rape" drugs. While alcohol, cannabis, benzodiazepines and stimulants are for the most part identified more frequently than GHB in drug-facilitated sexual assaults (DFSA), GHB is reported in up to $4.9 \%$ of DFSA. ${ }^{16}$

\section{Regulatory Framework and Therapeutic Use}

In acknowledgement of its highly addictive properties and significant abuse potential, GHB has been listed as a Schedule IV drug under the 1971 Convention on Psychotropic Substances by the United Nations Commission on Narcotic Drugs since 2001. ${ }^{5}$ Therapeutic use of GHB is currently limited to select settings. The sodium salt of GHB, sodium oxybate, is a controlled substance approved and monitored by the European Medicines Agency ${ }^{5,17}$ and the United States Food and 
Drug Administration for the treatment of narcolepsy and cataplexy. ${ }^{18}$ Sodium oxybate is also used in Italy and Austria for use in the management of alcohol withdrawal and relapse prevention in alcohol dependence. ${ }^{19}$

Other than alcohol withdrawal management, ${ }^{20}$ the use of pharmaceutical GHB has been investigated in GHB withdrawal with some promise, ${ }^{21,22}$ and is now a common treatment for GHB withdrawal in the Netherlands. ${ }^{23}$ Older studies have included use in opioid withdrawal where limited studies have shown modest benefit, ${ }^{24,25}$ and obstetric anesthesia, ${ }^{26}$ but these have not resulted in incorporation of GHB into practice. Therapeutic use of GHB or sodium oxybate is not currently approved by the Australian Therapeutic Goods Administration.

GBL and 1,4-BD are industrial solvents and are used in many industries including the production of automotive, textiles, electronics, pharmaceuticals, and polymers. In recognition of potential harms, the WHO has recommended that GBL and 1,4-BD are placed in Schedule 1 of the international convention. However, a number of member states have argued that control would place an unacceptable barrier to the industrial applications of these chemicals. Therefore, GBL and 1,4-BD are not under international control, but individual jurisdictions have a variety of control measures in place to prevent diversion from industrial uses. ${ }^{27}$

\section{Epidemiology of Current Use}

The 2021 World Drug Report from the United Nations Office on Drugs and Crime noted that in 2019, GHB consisted most of the sedatives and tranquilizers seized, exceeding seizures of benzodiazepines and barbiturates. ${ }^{28}$ In the same year, Sweden, the United States, New Zealand, Canada, China, and Australia were the six countries that recorded the largest quantities of GHB intercepted. ${ }^{28}$

In Australia, the National Drug Strategy Household Survey estimated a consistent $0.1 \%$ of the surveyed population above age 14 reported use of GHB within the previous 12 months both in 2016 and $2019 .{ }^{29}$ This has been stable over the previous 15 years. Age of initiation is somewhat later than for many substances, with most use initiated in the mid-20s. ${ }^{29}$ A similar population survey in the USA, the National Survey on Drug Use and Health, estimates the lifetime use of GHB in those aged over 18 years as $0.5 \%{ }^{30}$ European data indicate similarly low prevalence of GHB use. ${ }^{31}$ Some jurisdictions have higher use, with the Netherlands reporting past year use of $0.4 \%$ in $2018 .{ }^{32}$ In the United Kingdom, data from a number of sources including drug treatment and poisoning databases indicate an increase in GHB use up to 2015 that has now plateaued. Use and harms are seen predominantly in London. Very limited or no harms and presentations for treatment are reported from Scotland, Wales or Northern Ireland. ${ }^{33}$ Lack of granularity about GHB, GBL or 1,4-BD use in many national surveys limits the understanding of the epidemiology of use.

Some population subgroups have higher prevalence of GHB use. In a 2018 prospective Australian study of gay and bisexual men, close to $20 \%$ reported lifetime GHB use and 5.4\% reported use in the last six months. ${ }^{34}$ Data from the United Kingdom showed that in people presenting for substance use treatment, $22 \%$ of men who have sex with men reported problematic GBL use, in comparison to $2 \%$ of the heterosexual treatment presenters. ${ }^{35}$ The Australian National Illicit Drugs Survey Interview Report undertakes representative sampling across all states and territories annually. In the 2020 survey, of 884 people who inject drugs, one in ten reported use of GHB, GBL, or 1,4-BD within the past six months. ${ }^{36}$ Around $70 \%$ of people accessing treatment for GHB use report using multiple substances. Common coingestants are alcohol, methamphetamines, cannabis, cocaine, mephedrone and benzodiazepines. Ketamine, opioids and MDMA are less common co-ingestants, being reported by less than $20 \%$ of treatment seekers. ${ }^{2}$

Men are overrepresented in all studies of GHB use. ${ }^{2}$ In those presenting to emergency departments, young men predominate. ${ }^{2}$ Of these, $40 \%$ are also using alcohol or stimulants..$^{2}$ In community-based general population studies, men represent up to $90 \%$ of the sample. ${ }^{2}$ Up to $89 \%$ of people attending addiction treatment services for GHB use are men. ${ }^{2}$

Despite low prevalence of use compared to many other drugs, GHB is disproportionately identified in emergency presentations with drug toxicity. ${ }^{2}$ Acute harms of GHB can be noted in $10.6 \%$ of acute presentations with drug toxicity and up to a third (27\%) of critical care admissions from acute recreational drug toxicity in Euro-DEN Plus hospitals in 2019. ${ }^{31}$ Concerningly, in Australia, ambulance attendances where GHB toxicity is problematic have increased $147 \%$ between 2012 and 2019. ${ }^{37}$ 


\section{Clinical Features of GHB Use and Their Management}

Many people use GHB recreationally in a non-dependent fashion, and many may do so without adverse consequences. ${ }^{2}$ The clinical concerns regarding these drugs relate to harms of use that include toxicity, misadventure, dependency, and withdrawal. Its use in chemsex and risks relating to impairment of driving ability are also of concern.

\section{Acute Toxicity}

The narrow therapeutic index of GHB renders its use hazardous with poisoning or toxicity not uncommon with small titration of doses. Recreational use of GHB often occurs in settings of polydrug consumption, often with alcohol or stimulants. Recreational use of small doses of GHB may produce euphoric feelings and loss of inhibitions. Toxicity is dose-dependent; higher doses can lead to adverse effects such as headache, dizziness, nausea, vomiting, speech impairment, and anterograde amnesia. ${ }^{38}$ More severe acute toxicity or poisoning features can include respiratory depression, bradycardia, seizures, and reduced level of consciousness to deep coma. Death may result from severe respiratory compromise from aspiration, asphyxia or pulmonary oedema, or traumatic injury occurring as a result of sudden loss of consciousness. ${ }^{1}$ Co-ingestion of GHB or GBL with alcohol is associated with more severe toxicity, greater need for intensive care admission and more prolonged hospital admission. ${ }^{39}$

No effective antidote exists for the treatment of acute GHB poisoning, although early animal data utilising monocarboxylase transport inhibitors shows some promise. ${ }^{7}$ Treatment therefore consists primarily of observation and general supportive care. This may include ventilatory support depending on the severity of presentation. Often, recovery from acute poisoning can occur rapidly within hours as serum concentrations decrease given the short elimination half-life. The role of intubation in acute GHB toxicity is controversial. Two studies indicate that conservative airway management allows shorter length of stay compared to intubation and short-term outcomes are good. ${ }^{40,41}$ Decisions around intubation and invasive ventilation in this setting will clearly depend on recognition of GHB as the cause of impaired consciousness and local protocols around management of patients with impaired consciousness.

Overdose presentations are generally associated with recreational use. Risk factors for overdose include higher levels of GHB use, being male and polysubstance use. ${ }^{2}$ In the Illicit Drug Reporting System sentinel sample of those regularly injecting drugs and recent GHB use, 35\% reported non-fatal overdose in the last 12 months. ${ }^{42}$ Despite high rates of presentation to Emergency Departments relative to use, death as a result of GHB use is uncommon. In the 18 years to 2019, Australian coronial data identified 74 GHB-related deaths. ${ }^{1}$ In England and Wales, GHB-related deaths increased to 2015 but have plateaued since then, with 27 GHB-related deaths reported in $2018 .{ }^{33}$ However, the challenges of detecting GHB in post-mortem biological matrices may result in under-detection of GHB-related deaths. ${ }^{33}$ In the Australian death cohort, the majority were young men with unintentional overdose who died at home. ${ }^{1}$ Pulmonary oedema, acute pneumonia, and vomitus aspiration were common autopsy findings in these acute poisoning deaths. ${ }^{1}$ Perhaps, a more subtle consequence is that repeated GHB coma may be associated with impaired cognitive function and structural brain changes, although reversibility with GHB cessation has not yet been examined. ${ }^{43}$

\section{Chronic Use and Dependence}

Although GHB use disorder is not recognised in DSM-5, chronic recreational use of GHB can result in the development of tolerance, dependence, and a withdrawal syndrome. The criteria for the less specific diagnosis of Substance Use Disorder can often be met in this patient group. ${ }^{2}$ The lack of a recognition of GHB use disorder creates difficulties in defining its population prevalence. However, the number of people seeking treatment for GHB can be used as a proxy. US data indicate that GHB is identified as the drug in less than $0.05 \%$ of drug treatment episodes in $2019 .{ }^{44}$ In the United Kingdom, $0.2 \%$ of drug treatment episodes are for GHB use in 2017 and 2018, representing 450 to 500 people. ${ }^{33}$ The numbers in the Netherlands, with around a quarter of the population of the UK, are higher where 837 individuals received treatment for GHB use in 2015. ${ }^{32}$

Assessment of GHB use should include amount and frequency of use, and previous withdrawal experiences. Patterns of GHB use relevant to the DSM-5 criteria for substance use disorder relating to tolerance, loss of control, and physical 
and social harms should be explored. Co-ingestant use and other health problems should also be clarified, which can further assist with clinical risk stratifications. ${ }^{45}$

Tolerance can lead to dose escalation requirements, often by increasing dosing frequency due to its rapid absorption and elimination processes. It is common that those with GHB dependence require multiple, frequent doses throughout the day to avoid symptoms of withdrawal. Some authors report use every 1-3 hours in heavy users. ${ }^{46}$ Dependence can develop rapidly, with reports of development of severe GHB dependence within 12 months of initiation of use. ${ }^{47}$

\section{Withdrawal and Withdrawal Management}

GHB withdrawal features resemble that of alcohol withdrawal, but with more rapid onset of symptoms, often within hours of the last dose, given its short half-life, and can become severe rapidly. ${ }^{45}$ It is therefore imperative that treatment is commenced promptly and early to manage and prevent rapid deterioration of the withdrawal syndrome. GHB withdrawal features can include tremor, anxiety, agitation, sleep disruption, diaphoresis, and autonomic instability. Acute delirium, visual or auditory hallucinations and seizures have been reported in severe GHB withdrawal, and in some cases require intensive care management to avoid potentially fatal outcome. ${ }^{23,45,48}$ The more severe GHB withdrawal symptoms resolve within 2 weeks, but as with other sedative hypnotics, symptoms of insomnia, mood disruption and anxiety may persist for some time after the acute episode is complete. ${ }^{45,49}$

Factors proposed to predict development of withdrawal on cessation of use include amount and frequency of daily use (more than $4 \mathrm{~mL}$ daily, using more than 6 times per day), prior withdrawal, and using multiple substances. ${ }^{49}$ High level use of greater than $30 \mathrm{~mL}$ daily may be associated with risk of severe withdrawal. ${ }^{50}$

GHB withdrawal management has been undertaken in both ambulatory and in-patient settings. ${ }^{23,49-51}$ High rates of delirium are reported. ${ }^{23,49}$ Stratification based on level of GHB use and polysubstance use may help identify those likely to develop severe withdrawal who can be directed to in-patient withdrawal management. ${ }^{50}$ It may also be circumspect to have capacity for transfer to in-patient care should severe withdrawal develop in the ambulatory setting. ${ }^{49}$

The evidence base supporting medication management of withdrawal is limited and for the most part consists of case reports or case series. Most studies report initial management with benzodiazepines ${ }^{50,52,53}$ Outcomes from benzodiazepine alone can be suboptimal, particularly in those at high risk of severe withdrawal where delirium can emerge despite high doses of benzodiazepine. ${ }^{50,54}$ In response, a number of different medications have been added to benzodiazepine regimes. One small study of 19 patients initiated concomitant diazepam and baclofen in all patients with only 1 person developing a delirium requiring hospital admission. ${ }^{49}$ Other studies report benefit of initiating baclofen with benzodiazepine only in high-risk patients when severe withdrawal is anticipated. ${ }^{50}$ Other groups have advocated for initiating baclofen before commencing withdrawal. ${ }^{54}$ Despite the limited data supporting baclofen, UK guidelines have included a recommendation to initiate GHB withdrawal management with both baclofen and diazepam. ${ }^{55}$ The outcome of more robust trials is awaited. ${ }^{54}$

Pharmaceutical GHB has been more extensively studied than other medications in terms of patient numbers, although randomised controlled trials have not as yet been reported. ${ }^{21-23} \mathrm{~A}$ case series of 229 subjects indicated completion rates in acute withdrawal management of $85 \% .{ }^{22}$ A cohort study comparing pharmaceutical GHB to diazepam demonstrated that GHB was superior with respect to development of delirium and severity of withdrawal. ${ }^{23}$ In the Netherlands, GHB titration and tapering has acceptance as first-line therapy for GHB withdrawal. ${ }^{23}$ This is not the case in most other countries and the regulatory framework and cost may prove a barrier to use of sodium oxybate for this indication.

Multiple other pharmacological agents have been considered in GHB withdrawal. Case reports describe the addition of barbiturates to good effect after failure of benzodiazepine treatment. ${ }^{56-58} \mathrm{~A}$ recent case report describes a severe delirium resistant to benzodiazepine, baclofen and pharmaceutical GHB, which resolved after administration of intravenous then oral barbiturates. ${ }^{59}$ Other agents trialled with variable success include neuroleptics, ${ }^{51-53}$ anticonvulsants, ${ }^{53} \alpha 2$ adrenergic agonists (clonidine ${ }^{53}$ and dexmedetomidine ${ }^{48}$ ), and propofol, ${ }^{52}$ often after clinical deterioration. There is a clear need to trial and implement treatment regimens that will prevent this deterioration. 


\section{Relapse Prevention}

Optimal longer-term management of dependent GHB use is unknown. There is high (almost 70\%) risk of relapse post withdrawal. ${ }^{22}$ Data retrieved from the Dutch national drug treatment database indicate prolonged periods of treatment, frequent contact with services and high levels of repeat presentation for treatment in patients with GHB dependence. ${ }^{60}$ With regard to medications, an uncontrolled study of baclofen has shown some promise, but more rigorous trials evaluating its role in relapse prevention are needed. ${ }^{61}$

\section{Other Associated Problems}

In those attending for treatment of GHB use, a myriad of health and social problems are reported. Treatment seekers report problems with social functioning such as deteriorating work and educational performance, relationship difficulties and loss of driving license. ${ }^{49}$ Mental and physical health are problematic, with sleep disruption particularly problematic. These factors and GHB associated coma are all identified as reasons for seeking treatment. ${ }^{47,49}$

Chemsex describes the use of drugs to enhance, disinhibit or facilitate the sexual experience. ${ }^{35} \mathrm{GHB}$ and GBL are specifically listed as drugs commonly used in chemsex, along with methamphetamine and mephedrone. ${ }^{35}$ Challenges with chemsex include risky sexual behaviour with high rates of GHB use in people in Human Immunodeficiency Virus (HIV) treatment or presenting to sexual health clinics for post-exposure prophylaxis. ${ }^{35}$ Non-consensual sexual activity can be associated with chemsex. ${ }^{62}$ Death from unintentional overdose may also be seen. ${ }^{63}$ Progression from occasional use to more pervasive substance use disorder can occur. ${ }^{64}$ The lack of recognition of the association between sex and GHB use has meant that treatment was disjointed and therefore less effective. ${ }^{64}$ Culturally competent care recognising the association of chemsex and GHB use may improve treatment outcomes. ${ }^{64}$

In simulated driving tasks, subjects exposed to therapeutic doses of GHB as sodium oxybate had significant impairments at 1-hour post-dose. ${ }^{65}$ The impairments identified included simulations of collision and off-road accidents at 1-hour post-dose. ${ }^{65}$ Evaluation at 3- and 6-hours post-dose indicated gradual recovery from driving impairments over time as plasma concentrations fell, suggesting a concentration-dependent effect-impairment relationship. ${ }^{65}$ In an analysis of real-world GHB associated driving incidents, observed clinical signs included sedation, agitation, confusion, ataxia and dysarthria. ${ }^{6}$ Co-ingestion of GHB with other drugs in the impaired driver is common, predominantly with methamphetamine, but also cannabis, cocaine, and benzodiazepines. ${ }^{67}$ Therefore, warnings and increasing awareness regarding risks of driving impairment following GHB use is an important consideration for traffic safety messages and harm-minimisation strategies targeted for populations with high prevalence GHB use.

\section{Pharmacovigilance of Sodium Oxybate}

When monitoring prescription misuse of sodium oxybate, post-marketing studies reporting misuse or dependence are extremely rare in those without risk factors of psychiatric comorbidities or polydrug use history. ${ }^{68}$ However, greater consideration for controlled use or alternative therapy is recommended for "at risk populations". ${ }^{6}$

\section{Identifying GHB Use and Laboratory Analysis}

In the clinical setting, GHB use is generally identified through self-report and focussed questioning as described above. In acute toxicity, self-report is also important but in the unconscious patient, the ingestion may not be immediately clinically obvious. ${ }^{8}$ Drug testing for GHB is more typically undertaken in forensic circumstances rather than in the acute or treatment setting. The lack of inclusion in routine testing in the latter may in part be related to the challenges in its detection in assays. In the setting of acute toxicity, timely access to assays could improve management in complex cases. When not available acutely, sampling and appropriate storage with subsequent assay may assist in later review of the clinical scenario. ${ }^{8}$

The short half-life of GHB can result in under-detection. ${ }^{69}$ Therefore, timing of sample collection is important and should be done with vigilance, noting recommendations that whole blood samples be collected within eight hours and urine samples collected within twelve hours following known or suspected exposure. ${ }^{70}$ Hair and nail samples may be of use to detect GHB when faced with longer time delays to sampling. ${ }^{69}$ Assays for GHB are generally performed using 
highly sensitive and specific gas- or liquid-chromatography with tandem mass spectrometry or flame ionisation detection. ${ }^{69}$ Metabolomic profiling, the identification and quantification of multiple GHB metabolites, may prolong the detection window, but further investigation of this technique is needed. ${ }^{71}$

The interpretation of GHB concentrations is further compounded by its presence as an endogenous molecule, giving rise to the importance of ensuring appropriate cut-off concentrations in its reporting to avoid false-positive results. ${ }^{69}$ Further, certain storage conditions and time decay may lead to higher endogenous GHB concentrations due to accelerated catabolism of other molecules to GHB, particularly relevant in autopsy or post-mortem samples where re-distribution and putrefaction become problematic. ${ }^{69}$

The cut-off concentrations vary depending on the biological sample and timing at which it was obtained and analysed, with post-mortem cut-offs generally higher than ante-mortem samples cut-offs. Head hair sample analysis is a better matrix for documenting past exposure to GHB in cases where a significant time delay has occurred between the time of sampling and the time of exposure. ${ }^{72}$ The ratio of GHB concentrations in different segments of hair can allow identification of an acute exposure months after that exposure, although there is not consensus on the ratio that should be used. ${ }^{72}$ Recent advances in GHB detection include quantification of endogenous GHB in chest and pubic hair, ${ }^{73}$ and detection of exogenous GHB in these matrices awaits investigation. Other matrices of sampling exist, including saliva, vitreous humor, breast milk and cerebrospinal fluid. Some can be more stable in the post-mortem setting. ${ }^{69}$

\section{Conclusion and Future Directions}

Reports regarding GHB use prevalence are consistently lower compared to other illicit drugs. Given the relative ease of access and manufacture of GHB and its precursors, and the frequency of presentations to hospital, this may be an underrepresentation due to under-detection and under-reporting. Limitations in the methods used for epidemiological surveillance may contribute to under-estimation of prevalence of use. Some high-risk groups with unique treatment needs are seen and include men who have sex with men and those with polysubstance use. Despite the low reported prevalence of use, there are high levels of emergency department presentation with acute severe toxicity relative to other drugs. The consequences of acute poisoning include coma, hospitalisation, intensive care admission for airway support, and death. Other potential harms include driving impairment and at-risk sexual behaviour, along with the associated potential consequences.

Chronic dependence, tolerance, and withdrawal are seen, but are not recognised in DSM 5. Withdrawal can emerge quickly on cessation and be complicated by delirium, particularly in those with higher levels of use. Pharmacological treatment in GHB withdrawal is an area with an emerging body of evidence and includes benzodiazepines, baclofen, barbiturates and pharmaceutical GHB. High rates of relapse are seen after treatment for GHB use. Optimal relapse prevention strategies are unclear, but there is some recent consideration of baclofen use in maintenance of abstinence.

Future direction should focus on ensuring adequate coding for GHB use and harms to allow systematic data collection around the epidemiology of these. There is a need to improve both acute withdrawal and long-term management. The promising data from studies of pharmaceutical GHB should be explored in prospective randomised controlled trials. The role of baclofen and barbiturates in acute withdrawal management, and baclofen in relapse prevention needs clarification. The development and utility of improved analytical methods that facilitate detection in acute overdose, in the postmortem setting, after alleged assault and in longer-term treatment should be assessed. Development of interventions directed to specific sub-groups of users may improve treatment engagement and outcomes. Public health interventions about overdose recognition and response with high-risk groups should be assessed. Ongoing safety and harm reduction measures and discussions are important and should form a cornerstone of care in management of GHB use.

\section{Disclosure}

The authors report no conflicts of interest in this work.

\section{References}

1. Darke S, Peacock A, Duflou J, Farrell M, Lappin J. Characteristics and circumstances of death related to gamma hydroxybutyrate (GHB). Clin Toxicol. 2020;58(11):1028-1033. doi:10.1080/15563650.2020.1726378 
2. Dijkstra BAG, Beurmanjer H, Goudriaan AE, Schellekens AFA, Joosten EAG. Unity in diversity: a systematic review on the GHB using population. Int J Drug Policy. 2021;94:103230. doi:10.1016/j.drugpo.2021.103230

3. Snead OC 3rd, Gibson KM. Gamma-hydroxybutyric acid. N Engl J Med. 2005;352(26):2721-2732. doi:10.1056/NEJMra044047

4. Maitre M, Klein C, Mensah-Nyagan AG. Mechanisms for the specific properties of $\gamma$-Hydroxybutyrate in brain. Med Res Rev. 2016;36(3):363-388. doi:10.1002/med.21382

5. World Health Organization. Gamma-hydroxybutyric acid (GHB) critical review report ECoDD. WHO Thirty-fifth Meeting; June 4-8; 2012.

6. Elliott SP, Fais P. Further evidence for GHB naturally occurring in common non-alcoholic beverages. Forensic Sci Int. 2017;277:e36-e38. doi:10.1016/j.forsciint.2017.05.012

7. Felmlee MA, Morse BL, Morris ME. $\gamma$-Hydroxybutyric acid: pharmacokinetics, pharmacodynamics, and toxicology. AAPS J. 2021;23(1):22. doi:10.1208/s12248-020-00543-z

8. Stefani M, Roberts DM. 1,4-Butanediol overdose mimicking toxic alcohol exposure. Clin Toxicol. 2020;58(3):204-207. doi:10.1080/ 15563650.2019 .1617419

9. Absalom N, Eghorn LF, Villumsen IS, et al. $\alpha 4 \beta \delta$ GABA(A) receptors are high-affinity targets for $\gamma$-hydroxybutyric acid (GHB). Proc Natl Acad Sci U S A. 2012;109(33):13404-13409. doi:10.1073/pnas.1204376109

10. Abanades S, Farre M, Segura M, et al. Gamma-hydroxybutyrate (GHB) in humans: pharmacodynamics and pharmacokinetics. Ann N Y Acad Sci. 2006;1074:559-576. doi:10.1196/annals.1369.065

11. Liechti ME, Quednow BB, Liakoni E, et al. Pharmacokinetics and pharmacodynamics of gamma-hydroxybutyrate in healthy subjects. Br $J$ Clin Pharmacol. 2016;81(5):980-988. doi:10.1111/bcp.12863

12. Thai D, Dyer JE, Jacob P, Haller CA. Clinical pharmacology of 1,4-butanediol and gamma-hydroxybutyrate after oral 1,4-butanediol administration to healthy volunteers. Clin Pharmacol Ther. 2007;81(2):178-184. doi:10.1038/sj.clpt.6100037

13. Blumenfeld M, Suntay RG, Harmel,MH. Sodium gamma-hydroxybutyric acid: a new anesthetic adjuvant. Anesth Analg. 1962;41(6):721-726. doi:10.1213/00000539-196211000-00011

14. Rubin BAGN, Giarman NJ. The therapy of experimental influenza in mice with antibiotic lactones and related compounds. Yale J Biol Med. 1947;19(6):1017-1022.

15. Schep LJ, Knudsen K, Slaughter RJ, Vale JA, Mégarbane B. The clinical toxicology of $\gamma$-hydroxybutyrate, $\gamma$-butyrolactone and 1,4-butanediol. Clin Toxicol. 2012;50(6):458-470. doi:10.3109/15563650.2012.702218

16. Grela A, Gautam L, Cole MD. A multifactorial critical appraisal of substances found in drug facilitated sexual assault cases. Forensic Sci Int. 2018;292:50-60. doi:10.1016/j.forsciint.2018.08.034

17. European medicines agency. Avaialble from: https://www.ema.europa.eu/en/medicines/human/EPAR/xyrem. Accessed September $25,2021$.

18. United States Food and Drug Administration. Avaiailbe from: https://www.accessdata.fda.gov/drugsatfda_docs/label/2018/021196s030lbl.pdf. Accessed September 25, 2021.

19. Skala K, Caputo F, Mirijello A, et al. Sodium oxybate in the treatment of alcohol dependence: from the alcohol withdrawal syndrome to the alcohol relapse prevention. Expert Opin Pharmacother. 2014;15(2):245-257. doi:10.1517/14656566.2014.863278

20. Busardò FP, Kyriakou C, Napoletano S, Marinelli E, Zaami S. Clinical applications of sodium oxybate (GHB): from narcolepsy to alcohol withdrawal syndrome. Eur Rev Med Pharmacol Sci. 2015;19(23):4654-4663.

21. de Jong CA, Kamal R, Dijkstra BA, de Haan HA. Gamma-hydroxybutyrate detoxification by titration and tapering. Eur Addict Res. 2012;18(1):4045. doi:10.1159/000333022

22. Dijkstra BA, Kamal R, van Noorden MS, de Haan H, Loonen AJ, De Jong CA. Detoxification with titration and tapering in gamma-hydroxybutyrate (GHB) dependent patients: the Dutch GHB monitor project. Drug Alcohol Depend. 2017;170:164-173. doi:10.1016/j. drugalcdep.2016.11.014

23. Beurmanjer H, Luykx JJ, De Wilde B, et al. Tapering with pharmaceutical GHB or Benzodiazepines for Detoxification in GHB-dependent patients: a matched-subject observational study of treatment-as-usual in Belgium and The Netherlands. CNS Drugs. 2020;34(6):651-659. doi:10.1007/ s40263-020-00730-8

24. Gallimberti L, Schifano F, Forza G, Miconi L, Ferrara SD. Clinical efficacy of gamma-hydroxybutyric acid in treatment of opiate withdrawal. Eur Arch Psychiatry Clin Neurosci. 1994;244(3):113-114. doi:10.1007/BF02191883

25. Rosen MI, Pearsall HR, Woods SW, Kosten TR. The effect of gamma-hydroxybutyric acid on naloxone-precipitated opiate withdrawal. Neuropsychopharmacology. 1996;14(3):187-193. doi:10.1016/0893-133X(95)00087-T

26. Geldenhuys FG, Sonnendecker EW, De Klrk MC. Experience with sodium-gamma-4-hydroxybutyric acid (gamma-OH) in obstetrics. J Obstet Gynaecol Br Commonw. 1968;75(4):405-413. doi:10.1111/j.1471-0528.1968.tb00137.x

27. World. Health Organization Commission on Narcotic Drugs Fifty-eighth session. Note by the Secretariat on the scope of control of substances: proposed scheduling recommendations initiated by WHO; 2015. Available from: https://www.unodc.org/documents/commissions/CND/CND_ Sessions/CND_58/ECN72015_8_eV1501009.pdf. Accesed December 20, 2021.

28. World Drug Report. United Nations publication, Sales No. E.21.XI.8; 2021. Available from: https://www.unodc.org/unodc/en/data-and-analysis/ wdr2021.html. Accessed December 20, 2021.

29. Australian Institute of Health and Welfare. National Drug Strategy Household Survey. Statistics series no. 32. PHE 270; 2019. Available from: https://www.aihw.gov.au/reports/illicit-use-of-drugs/national-drug-strategy-household-survey-2019/contents/summary. Accessed December 20, 2021.

30. Center for behavioural health statistics and quality. Results from the 2019 national survey on drug use and health: detailed tables. Rockville, MD: Substance Abuse and Mental Health Services Administration; 2020 [cited December 20, 2021]. Avaialble from: https://www.samhsa.gov/data/. Accessed January 6, 2021.

31. European Monitoring Centre for Drugs and Drug Addiction. European drug report 2021: trends and developments. Luxembourg: Publications Office of the European Union; 2021.

32. WODC Repository. National drug monitor; 2020. Availalbe from: https://repository.wodc.nl/handle/20.500.12832/3035. Accessed December 20, 2021. 
33. Advisory Council on the Misuse of Drugs. An assessment of the harms of gamma-hydroxybutyric acid (GHB), gamma-butyrolactone (GBL), and closely related compounds. Available from: https:/www.gov.uk/government/publications/assessment-of-the-harms-of-gamma-hydroxybutyric-acidgamma-butyrolactone-and-closely-related-compounds. Accessed December 20, 2021.

34. Hammoud MA, Bourne A, Maher L, et al. Intensive sex partying with gamma-hydroxybutyrate: factors associated with using gamma-hydroxybutyrate for chemsex among Australian gay and bisexual men - results from the Flux Study. Sex Health. 2018;15(2):123-134. doi:10.1071/ SH17146

35. Edmundson C, Heinsbroek E, Glass R, et al. Sexualised drug use in the United Kingdom (UK): a review of the literature. Int J Drug Policy. 2018;55:131-148. doi:10.1016/j.drugpo.2018.02.002

36. Peacock AU, Karlsson A, Price O, et al. Australian drug trends 2020: key findings from the Illicit Drug Reporting System (IDRS) interviews. Available from: https://ndarc.med.unsw.edu.au/resource/australian-drug-trends-2020-key-findings-national-illicit-drug-reporting-system-idrs. Accessed December 20, 2021.

37. Arunogiri S, Moayeri F, Crossin R, et al. Trends in gamma-hydroxybutyrate-related harms based on ambulance attendances from 2012 to 2018 in Victoria, Australia. Addiction. 2020;115(3):473-479.

38. Andresen H, Aydin BE, Mueller A, Iwersen-Bergmann S. An overview of gamma-hydroxybutyric acid: pharmacodynamics, pharmacokinetics, toxic effects, addiction, analytical methods, and interpretation of results. Drug Test Anal. 2011;3(9):560-568. doi:10.1002/dta.254

39. Galicia M, Dargan PI, Dines AM, et al. Clinical relevance of ethanol coingestion in patients with GHB/GBL intoxication. Toxicol Lett. 2019;314:37-42. doi:10.1016/j.toxlet.2019.07.001

40. Dietze P, Horyniak D, Agius P, et al. Effect of intubation for gamma-hydroxybutyric acid overdose on emergency department length of stay and hospital admission. Acad Emerg Med. 2014;21(11):1226-1231. doi:10.1111/acem.12516

41. van Helmond L, Gresnigt FMJ. Safety of withholding intubation in gamma-hydroxybutyrate- and gamma-butyrolactone-intoxicated coma patients in the emergency department. Eur J Emerg Med. 2020;27(3):223-227. doi:10.1097/MEJ.0000000000000649

42. Karlsson ASR, Peacock A Use of GHB amongst sentinel samples of people who regularly use illicit drugs in Adelaide, South Australia, 2020. Drug Trends Bulletin Series. Sydney: National Drug and Alcohol Research Centre, UNSW Sydney; 2021. Available from: https:/ndarc.med.unsw.edu. au/sites/default/files/ndarc/resources/South\%20Australia\%20GHB\%20Use\%20FINAL.pdf. Accessed December $20,2021$.

43. Amsterdam JV, Brunt TM, Pereira FR, Crunelle CL, Brink WVD. Cognitive impairment following clinical or recreational use of gammahydroxybutyric acid (GHB). A systematic review. Curr Neuropharmacol. 2021. doi:10.2174/1570159X19666210610094352

44. Substance Abuse and Mental Health Services Administration, Center for Behavioral Health Statistics and Quality. Treatment Episode Data Set (TEDS): 2019. Admissions to and discharges from publicly funded substance use treatment. Rockville, MD: Substance Abuse and Mental Health Services Administration; 2021. Available from: https://www.samhsa.gov/data/sites/default/files/reports/rpt35314/2019_TEDS_Proof.pdf. Accessed December 8, 2021.

45. Phan V, Arunogiri S, Lubman D. The assessment and management of gamma hydroxybutyrate use in general practice. Aust $J$ Gen Pract. 2020;49:73-78. doi:10.31128/AJGP-05-19-4953

46. Durgahee S, Allen G, Williams H. The “G” men: a profile of GBL/GHB users in an area of high drug-related mortality. Ir J Psychol Med. 2014;31 (4):275-280. doi:10.1017/ipm.2014.39

47. Brunt TM, Koeter MW, Hertoghs N, van Noorden MS, Van den brink W. Sociodemographic and substance use characteristics of gamma hydroxybutyrate (GHB) dependent inpatients and associations with dependence severity. Drug Alcohol Depend. 2013;131(3):316-319. doi:10.1016/j.drugalcdep.2012.12.023

48. Tay EM, Graham RI, Day RO. Severe GHB withdrawal delirium managed with dexmedetomidine. Med J Aust. 2016;205(6):251-252. doi:10.5694/ mja16.00269

49. Bell J, Collins R. Gamma-butyrolactone (GBL) dependence and withdrawal. Addiction. 2011;106(2):442-447. doi:10.1111/j.13600443.2010.03145.x

50. McDonough M. GHB dependence: lessons from a small case series. J Alcohol Drug Depend. 2013;112. doi:10.4172/2329-6488.1000112

51. Cappetta M, Murnion BP. Inpatient management of gamma-hydroxybutyrate withdrawal. Australas Psychiatry. 2019;27(3):284-287. doi:10.1177/ 1039856218822748

52. Dyer JE, Roth B, Hyma BA. Gamma-hydroxybutyrate withdrawal syndrome. Ann Emerg Med. 2001;37(2):147-153. doi:10.1067/ mem.2001.112985

53. Kamal RM, van Noorden MS, Wannet W, Beurmanjer H, Dijkstra BA, Schellekens A. Pharmacological treatment in gamma-Hydroxybutyrate (GHB) and gamma-Butyrolactone (GBL) dependence: detoxification and relapse prevention. CNS Drugs. 2017;31(1):51-64. doi:10.1007/s40263016-0402-z

54. Lingford-Hughes A, Patel Y, Bowden-Jones O, et al. Improving GHB withdrawal with baclofen: study protocol for a feasibility study for a randomised controlled trial. Trials. 2016;17(1):472. doi:10.1186/s13063-016-1593-9

55. Abdulrahim D, Bowden-Jones O; on behalf of the NEPTUNE Expert Group. Guidance on the management of acute and chronic harms of club drugs and novel psychoactive substances. novel psychoactive treatment UK network (Neptune). London; 2015. Available from: http://neptuneclinical-guidance.co.uk/wp-content/uploads/2015/03/NEPTUNE-Guidance-March-2015.pdf. Accessed December 20, 2021.

56. Sivilotti ML, Burns MJ, Aaron CK, Greenberg MJ. Pentobarbital for severe gamma-butyrolactone withdrawal. Ann Emerg Med. 2001;38(6):660665. doi:10.1067/mem.2001.119454

57. Ghio L, Cervetti A, Respino M, Belvederi Murri M, Amore M. Management and treatment of gamma butyrolactone withdrawal syndrome: a case report and review. J Psychiatr Pract. 2014;20(4):294-300. doi:10.1097/01.pra.0000452567.84825.07

58. McDonough M, Kennedy N, Glasper A, Bearn J. Clinical features and management of gamma-hydroxybutyrate (GHB) withdrawal: a review. Drug Alcohol Depend. 2004;75(1):3-9. doi:10.1016/j.drugalcdep.2004.01.012

59. Vos CF, Pop-Purceleanu M, van den Berg MJW, Schellekens AFA. Successful treatment of severe, treatment resistant GHB withdrawal through thiopental-coma. Subst Abus. 2021;42(1):33-38. doi:10.1080/08897077.2020.1827124

60. van Noorden MS, Mol T, Wisselink J, Kuijpers W, Dijkstra BAG. Treatment consumption and treatment re-enrollment in GHB-dependent patients in The Netherlands. Drug Alcohol Depend. 2017;176:96-101. doi:10.1016/j.drugalcdep.2017.02.026

61. Beurmanjer H, Kamal RM, de Jong CAJ, Dijkstra BAG, Schellekens AFA. Baclofen to prevent relapse in Gamma-Hydroxybutyrate (GHB)dependent patients: a multicentre, open-label, non-randomized, controlled trial. CNS Drugs. 2018;32(5):437-442. doi:10.1007/s40263-018-0516-6 
62. Drückler S, Speulman J, van Rooijen M, De Vries HJC. Sexual consent and chemsex: a quantitative study on sexualised drug use and nonconsensual sex among men who have sex with men in Amsterdam, the Netherlands. Sex Transm Infect. 2021;97(4):268-275. doi:10.1136/sextrans2020-054840

63. Hockenhull J, Murphy KG, Paterson S. An observed rise in $\gamma$-hydroxybutyrate-associated deaths in London: evidence to suggest a possible link with concomitant rise in chemsex. Forensic Sci Int. 2017;270:93-97. doi:10.1016/j.forsciint.2016.11.039

64. Stuart D. Chemsex: origins of the word, a history of the phenomenon and a respect to the culture. Drugs Alcohol Today. 2019;19(1):3-10. doi:10.1108/DAT-10-2018-0058

65. Liakoni E, Dempsey DA, Meyers M, et al. Effect of $\gamma$-hydroxybutyrate (GHB) on driving as measured by a driving simulator. Psychopharmacology. 2018;235(11):3223-3232. doi:10.1007/s00213-018-5025-2

66. Jones AW, Holmgren A, Kugelberg FC. Driving under the influence of gamma-hydroxybutyrate (GHB). Forensic Sci Med Pathol. 2008;4(4):205211. doi:10.1007/s12024-008-9040-1

67. Griffiths A, Hadley L. The prevalence of gamma-hydroxybutyrate (GHB) in motor vehicle drivers and its co-administration with amphetamine type substances (ATS) in Queensland, Australia (2011-2018). Forensic Sci Int. 2019;303:109958. doi:10.1016/j.forsciint.2019.109958

68. Addolorato G, Lesch OM, Maremmani I, et al. Post-marketing and clinical safety experience with sodium oxybate for the treatment of alcohol withdrawal syndrome and maintenance of abstinence in alcohol-dependent subjects. Expert Opin Drug Saf. 2020;19(2):159-166. doi:10.1080/ 14740338.2020 .1709821

69. Busardò FP, Jones AW. Interpreting $\gamma$-hydroxybutyrate concentrations for clinical and forensic purposes. Clin Toxicol. 2019;57(3):149-163. doi:10.1080/15563650.2018.1519194

70. Busardò FP, Varì MR, Di Trana A, Malaca S, Carlier J, Di Luca NM. Drug-facilitated sexual assaults (DFSA): a serious underestimated issue. Eur Rev Med Pharmacol Sci. 2019;23(24):10577-10587. doi:10.26355/eurrev_201912_19753

71. Steuer AE, Raeber J, Simbuerger F, et al. Towards extending the detection window of gamma-hydroxybutyric acid-an untargeted metabolomics study in serum and urine following controlled administration in healthy men. Metabolites. 2021;11(3). doi:10.3390/metabo11030166

72. Busardò FP, Pichini S, Zaami S, Pacifici R, Kintz P. Hair testing of GHB: an everlasting issue in forensic toxicology. Clin Chem Lab Med. 2018;56 (2):198-208. doi:10.1515/cclm-2017-0397

73. Bertol E, Mari F, Lachi A, Tespio G, Vaiano F. Determination of endogenous GHB levels in chest and pubic hair. Forensic Sci Int. 2021;325:110857. doi:10.1016/j.forsciint.2021.110857

\section{Publish your work in this journal}

Substance Abuse and Rehabilitation is an international, peer-reviewed, open access journal publishing original research, case reports, editorials, reviews and commentaries on all areas of addiction and substance abuse and options for treatment and rehabilitation. The manuscript management system is completely online and includes a very quick and fair peer-review system. Visit http://www.dovepress.com/testimonials.php to read real quotes from published authors.

Submit your manuscript here: http://www.dovepress.com/substance-abuse-and-rehabilitation-journal 